\title{
Diferencias morfológicas en el copepodo Tropocyclops prasinus
}

\author{
Tecla Riera ${ }^{1}$; Marta Estrada ${ }^{2}$
}

1 Departamento de Ecología. Universidad de Barcelona

2 Instituto de Investigaciones Pesqueras, Barcelona

\section{SUMMARY}

MORPHOLOGICAL DIFFERENTIATION IN THE COPEPOD Tropocyclops prasinus.

Populations of Tropocyclops prasinus (Copepoda Ciclopidae) of different origins present a marked morphological variability. To analize this variability, sets of fifty individuals were obtained in two seasons of the year from 15 reservoirs scattered all over Spain. Twenty-two measurements were taken of each organism and a principal component analysis of those data was carried out.

The first principal component was positively correlated with all the variables and indicated overall size variability. The second and third components reflected variations of shape. The variables corresponding to particular organs of the body fell together in the space generated by the components; their distribution suggested the existence of morphogenetic gradients. Geographically close populations tend to present bimetric similarities. This finding could have a genetical basis or could be associated with similar environmental conditions.

\section{INTRODUCCION}

La morfología de los individuos de una especie presenta variaciones que se ponen de manifiesto cuando se comparan individuos de una misma edad de poblaciones diferentes. La variación de las dimensiones de los organismos, puede ser debida tanto a la influencia directa del ambiente como al resultado de la selección de combinaciones genéticas (MARGALEF, 1955). Por regla general los individuos de una misma especie desarrollados bajo diferentes temperaturas tienen distinto tamaño de manera que los criados a temperatura más alta son menores. MARGALEF (1955) presenta muchos ejemplos sobre la variación de las dimensiones de los organismos en relación con la temperatura.

Algunos organismos como rotiferos y cladóceros presentan variaciones estacionales de tamaño muy evidentes y espectaculares en algunas partes del cuerpo con un crecimiento alométrico muy marcado (BROOKS, - 1947; MARGALEF, 1947, 1955, 1974; HUTCHINSON, 1967). En los copépodos estas variaciones de tamaño se conocen desde hace tiempo (LILLJEBORG, 1901 


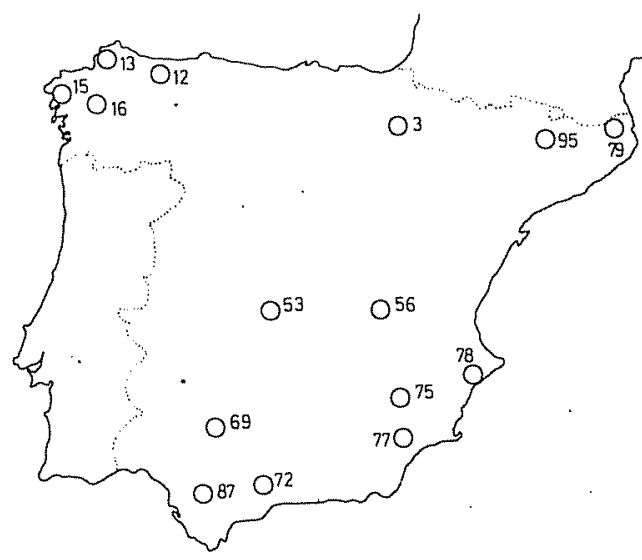

Figura 1. Localización de los embalses estudiados. 3 Alloz; 12 Arbon; 13 Forcadas; 15 Fervenza; 16 Porto de Mouros; 53 Guajaraz; 56 Alarcón; 69 Retortillo; 72 Conde de Guadalhorce; 75 Cenajo; 77 Puentes; 78 Amadorio; 79 Boadella; 87 Bornos;95 Oliana. Más información en MARGALIF et al. 1976.

citado por TONOLLI, 1949), pero son menos evidentes, y su estudio requiere un análisis biométrico de diferentes partes del organismo (SEWELL, 1948; TONOLLI, 1949, 1961 entre otros).

En este estudio se presenta un análisis biométrico detallado, en el que se intenta profundizar en el significado de las variaciones morfológicas en copépodos. Se realiza un análisis de asociación de caracteres que relaciona de modo cuantitativo diversas medidas del organismo. Desde un punto de vista morfogenético y evolutivo estas relaciones son muy importantes, ya que es interesante averiguar si algunas partes del cuerpo, por ejemplo las asociadas a la alimentación, natación o otras funciones orgánicas presentan un tipo de variación particular, dentro de la variación general del organismo. Por otra parte también interesa ver si las diferencias entre poblaciones tienen una base ecológica.

El estudio se ha llevado a cabo con Tropocy'clops prasinus (copépodo ciclópido cosmopolita de agua dulce) de la que desde hace tiempo se conocen variaciones

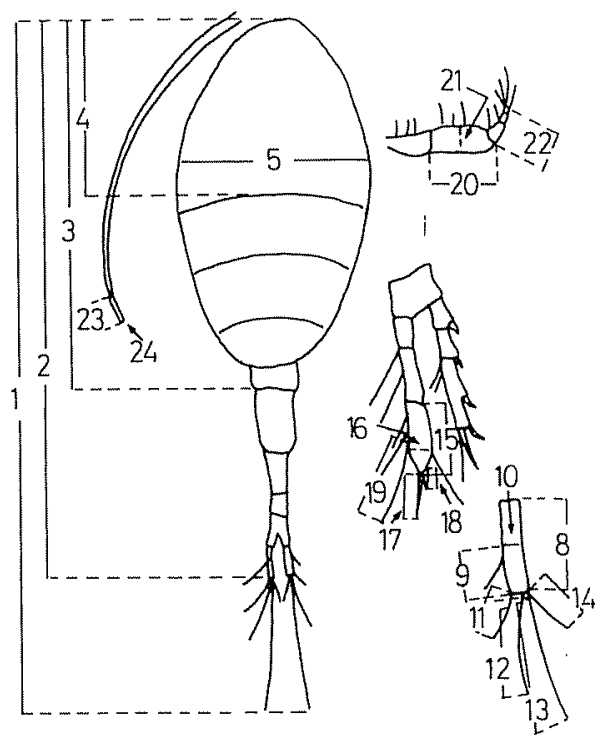

Figura 2. Situación de los caracteres seleccionados como variables. 1 Longitud total del organismo. 2 Longitud total del organismo sin las sedas de la furca. 3 Longitud del cefalotórax. 4 Longitud del primer segmento del cefalotórax. 5 Anchura del cefalotórax. 8 Longitud total de la furca. 9 Longitud de la parte terminal de la furca (a partir de la seda). 10 Anchura de la furca. 11 Longitud de la seda externa, 12 Longitud de la seda mediana externa, 13 Longitud de la seda mediana intorna. 14 Longitud de la seda interna. 15 Longitud del tercer artejo de el cndopodito de la $4^{2}$ pata. 16 Anchura del tercer artejo del endopodito de la $4^{a}$ pata. 17 Longitud de la espina interna del tercer artejo del endopodito de la $4^{\mathrm{a}}$ pata. 18 Longitud de la espina intema del tercer artejo del endopodito de la 4 a pata. 19 Longitud de la seda interna del tercer artejo del endopodito de la $4^{a}$ pata. 20 Longitud del segundo segmento del maxilipedo. 21 Anchiura del segundo segmento del maxilipedo. 22 Longitud de los segmentos terminales del maxilípedo. 23 Longitud del último artejo de la antena. 24 Anchura del último artẹjo de la antena.

de tamaño en organismos procedentes de diferentes localidades o de distintas épocas del año (MARGALEF, 1950; MIRACLE, 1976). 


\section{MATERIAL Y METODOS}

Se han estudiado copépodos recolectados en dos épocas del año en 15 embalses situados en diversos puntos de la Península Ibérica (fig. 1). Las muestras pertenecen al Departamento de Ecología y proceden del estudio de Embalses llevado a cabo por un equipo de investigadores de dicho Departamento. Se estudiaron aquellos embalses, en los que la abundancia de Tropocyclops prasinus, era superior a un $5 \%$ del total de crustáceos, en las muestras procedentes de la 1a Campaña (otoño-invierno de 1972-73). En algunos de los embalses como Fervenza (15), Alarcón (56) y Amadorio (78), sólo se han podido estudiar los organismos correspondientes a esta época ya que en la $2^{\text {a }}$ Campaña (verano de 1973) habian disminuido considerablemente debido a la variabilidad propia de la densidad de la especie durante el ciclo anual.

Las muestras fueron tomadas por medio de una red cónica de $0,75 \mathrm{~m}$. de diámetro, $1,20 \mathrm{~m}$. de longitud y 250 micras de abertura de malla. Para cualquier referencia a cómo se han tomado las muestras, situación del punto de muestreo en el embalse y resultados de los analísis químicos, físicos y biológicos veáse MARGALEF et al. (1976).

De cada muestra se separaron 50 individuos (hembras) y se tomaron en cada una 22 medidas de distintas partes del cuerpo (fig. 2). Para una explicación más detallada de los métodos utilizados y de las razones por las que se seleccionaron estas medidas como variables veáse RIERA (1981). Para poner de manifiesto las variaciones de tamaño de los organismos, se han calculado las medias y errores de la media de cada una de las variables medidas en cada localidad y época del año (veáse el apéndice).

A fin de obtener una descripción de las tendencias más importantes de la variabilidad total de los datos, se ha realizado un analisis de componentes principales. El análisis se hizo sobre la matriz de correlaciones a la que no se aplicó ninguna rotación. No se utilizó la matriz de covarianzas porque aunque trataba de medidas comparables, no todas tenian el mismo ta- maño $\mathrm{y}$ en estos casos la utilización de esta matriz presenta problemas ya que los valores de las variables de mayor tamaño influyen demasiado en el resultado (SEAL, 1964; CUADRAS, 1979). Los datos se transformaron logaritmicamente $(\mathrm{x} \longrightarrow \ln (\mathrm{x}))$. El valor de las componentes (score) $Y_{j}$ se ha calculado según la fórmula de COOLEY \& LOHNES (1971):

$$
Y_{j}=\sum_{i=1}^{n} a_{i j} \frac{x_{i}-\bar{x}_{i}}{s_{i}} \frac{1}{\lambda_{j}}
$$

$\mathrm{Y}_{\mathrm{j}}=$ Valor (score) del componente $\mathrm{j}$

$\mathrm{a}_{\mathrm{ij}}=$ (loading) del componente $\mathrm{j}$ para la especie i, que equivale en este caso al coeficiente de correlación de componente $j$ con la especie $i$.

$\mathrm{x}_{\mathrm{i}}=$ logaritmo neperiano de la variable $\mathrm{i}$

$\bar{x}_{i}=$ media de $x_{i}$

$s_{i}=$ desviación típica de $x_{i}$

$\lambda_{j}=$ valor propio del componente $j$

Los cálculos se han llevado a cabo con el IBM 1.130 del Instituto de Investigaciones Pesqueras de Barcelona, y se han utilizado las subrutinas del SSP de IBM (IBM, 1969). Con los datos se han llevado a cabo dos análisis (tabla I). En uno de ellos (a) se utilizaron como observaciones el conjunto de medidas de las 22 variables para cada uno de los individuos (1.192 casos); en el otro (b) se utilizó el conjunto de medias de cada variable para cada localidad y época del año (27 casos).

\section{RESULTADOS Y DISCUSION}

La observación de los resultados expuestos en el apéndice pone de manifiesto que por un lado existen variaciones de tamaño de las diferentes partes del cuerpo según la localidad de la que proceden las muestras, mientras que por otro lado existen diferencias de tamaño en una misma localidad según la época del año en que se han tomado las muestras.

A pesar de que las muestras proceden de un área limitada (la peninsula Ibérica), encontramos un rango de variación de la lon- 
TABLA 1. Tropocyclos prasinus (hembra). Coeficientes de carga de las variables (que equivalen en este caso a los coeficientes de correlación), con los tres primeros componentes. (a) análisis realizado con todos los individuos (b) análisis realizado con las medias de las variables. Para la identificación de las varia. bles ver fig. 2 .

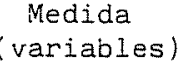

Medida
(variables)

1
2
3
4
5
8
9
10
11
12
13
14
15
16
17
18
19
20
21
22
23
24

\begin{tabular}{crr}
\hline 1 & \multicolumn{1}{c}{2} & \multicolumn{1}{c}{3} \\
& & \\
0,939 & $-0,016$ & $-0,147$ \\
0,892 & $-0,186$ & $-0,184$ \\
0,765 & $-0,239$ & $-0,183$ \\
0,819 & $-0,239$ & $-0,129$ \\
0,831 & $-0,187$ & $-0,126$ \\
0,886 & 0,095 & $-0,030$ \\
0,754 & $-0,082$ & $-0,104$ \\
$0,5-2$ & $-0,531$ & 0,140 \\
0,832 & 0,004 & $-0,164$ \\
0,854 & 0,196 & $-0,124$ \\
0,820 & 0,426 & 0,024 \\
0,586 & $-0,313$ & $-0,305$ \\
0,836 & 0,153 & $-0,097$ \\
0,574 & $-0,203$ & 0,498 \\
0,787 & $-0,077$ & $-0,163$ \\
0,666 & 0,001 & 0,047 \\
0,770 & 0,494 & 0,044 \\
0,771 & 0,261 & 0,132 \\
0,661 & $-0,011$ & 0,431 \\
0,576 & 0,146 & 0,358 \\
0,806 & 0,250 & 0,114 \\
0,457 & $-0,327$ & 0,573
\end{tabular}

\begin{tabular}{ccc}
\hline 1 & 2 & \multicolumn{1}{c}{3} \\
& & \\
0,955 & $-0,002$ & $-0,043$ \\
0,902 & $-0,193$ & $-0,115$ \\
0,788 & $-0,321$ & $-0,280$ \\
0,921 & $-0,219$ & $-0,084$ \\
0,906 & $-0,169$ & $-0,074$ \\
0,967 & 0,123 & $-0,053$ \\
0,944 & $-0,122$ & 0,102 \\
0,563 & $-0,553$ & $-0,088$ \\
0,381 & $-0,162$ & $-0,182$ \\
0,914 & 0,111 & 0,368 \\
0,365 & 0,439 & 0,084 \\
0,613 & $-0,180$ & 0,541 \\
0,909 & 0,102 & 0,244 \\
0,610 & 0,003 & $-0,376$ \\
0,373 & $-0,216$ & 0,380 \\
0,305 & 0,008 & 0,065 \\
0,811 & 0,532 & 0,095 \\
0,911 & 0,343 & 0,032 \\
0,930 & 0,145 & 0,045 \\
0,393 & 0,245 & $-0,073$ \\
0,374 & 0,350 & $-0,279$ \\
0,704 & $-0,303$ & $-0,279$
\end{tabular}

gitud total del organismo sin sedas de la furca (variable 2, fig. 2) bastante amplio, desde 0,686 $\mathrm{mm}$. (media en el embalse de Buadella, 79) a 0,841 (media del embalse de Forcadas, 13). Otros autores (GURNEY, 1933: MARGALEF, 1953; DUSSART, 1969) hallan también que la longitud de estos organismos varia entre 0,6 y $0,9 \mathrm{~mm}$. Al estudiar los organismos de una misma localidad en épocas del año distintas, encontramos resultados semejantes a los hallados por otros autores (MARGALEF, 1950; MIRACLE, 1976); los organismos que se midieron procedentes de muestras tomadas en invicrno, son de mayor tamaño que los que se recolectaron en verano y en la mayoría de los casos existe una relación inversa entre tamaño y temperatura (fig. 3); con algunas excepciones, en los embalses de Alloz (3); Retortillo (69) y Cenajo (75). En estas localidades la diferencia de tenperatura entre las dos campañas, bastante próxinas en el tiempo, era muy pequeña, por lo que probablemente no hubo suficiente tiempo para un cambio significativo en las medidas de los organismos. Por otro lado debe tenerse en cuenta que los individuos adultos capturados en unas condiciones ambientales determinadas pertenecen a una generación nacida unas semanas antes y que por consiguiente ha podido estar sometida ( $y$ normalmente 


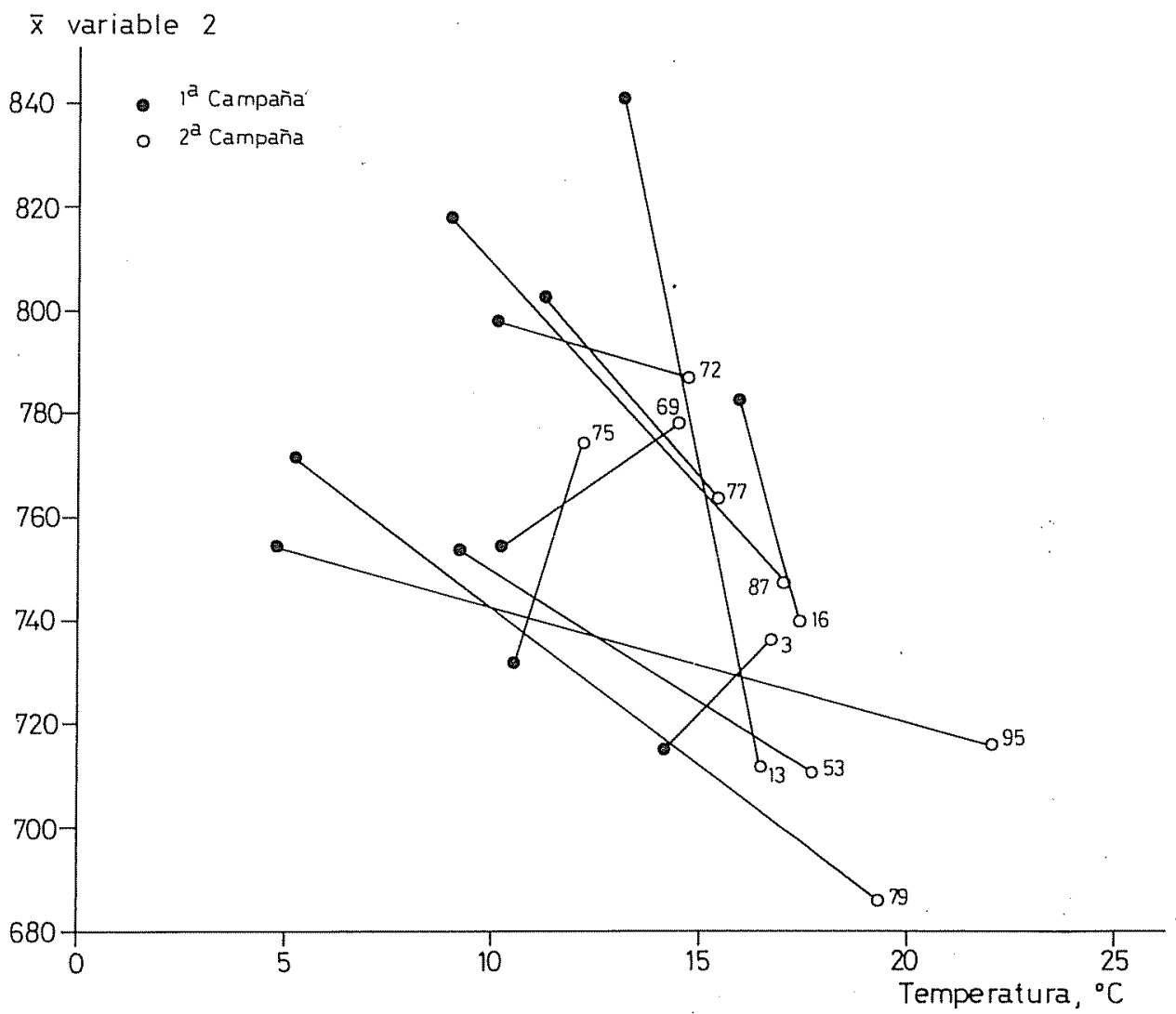

Figura 3. Tropocy clops prasinus (hembras). Relación de la variable 2 (longitud total sin sedas) con la media ponderada de la temperatura.

lo ha estado) a condiciones ambientales distintas de las del momento del muestreo. Esta es la explicación más palusible a la relación directa de temperatura y tamaño que parece deducirse en la figura 3 para estos embalses.

Las varianzas explicadas por los tres primeros componentes, en el análisis realizado con el conjunto de medidas de todos los datos (a en la tabla I) son respectivamente del $57,11 \%, 6,42 \%$ y $5,69 \%$. En la tabla I se pueden ver los valores de los "loadings" o coeficientes de carga de las variables para los tres pimeros componentes.
Los coeficientes de carga con el primer componente, que explica una gran parte de la varianza, son siempre positivos e indican la asociación de éste con la variación general de tamaño del organismo; como se ve, los valores más altos de estos coeficientes de carga corresponden a las variables de rnayor tamaño. En el caso del $3^{\mathrm{cr}}$ componente, los valores más elevados corresponden a medidas transversales. En general estos resultados son comparables a los obtenidos por otros autores en estudios biométricos de diversos organismos (JOLICOEUR \& MOSSIMAN, 1960; REYMENT \& SANDBERG, 1963; SEAL, 


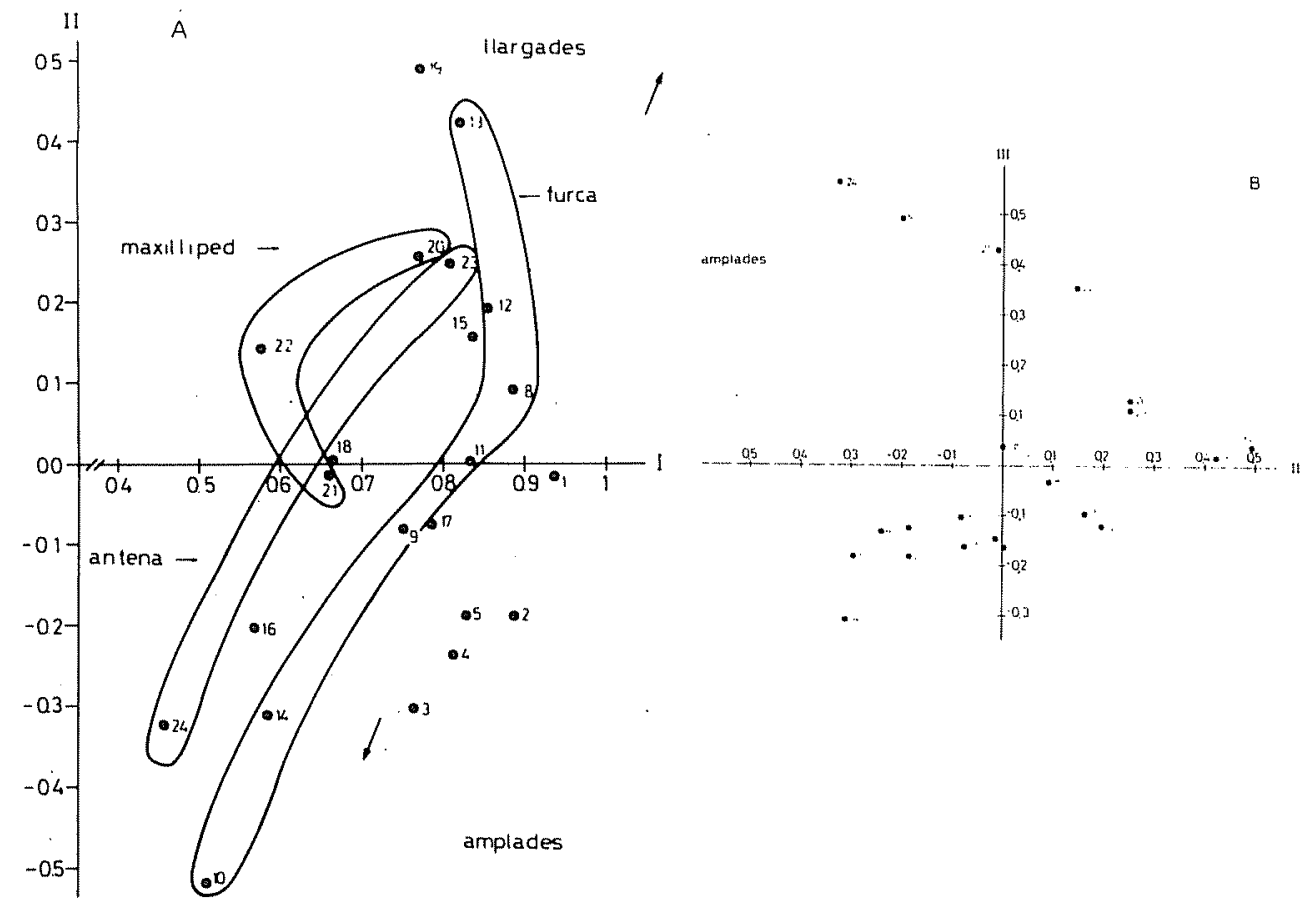

Figura 4. Tropocyclops prasinus (hembra). Análisis de componentes principales. A) Representación de las variables en el espacio de los dos primeros componentes. Las variables correspondientes a un mismo ór gano se han agrupado. B) Representación de las variables en el espacio del $2^{\circ}$ y $3^{\text {er }}$ componentes

1964; REYMENT, 1966 entre otros). Por todo ello se puede concluir que el primer componente, que está correlacionado positivamente con todas las variables, explica una parte importante de la varianza y se asocia al tamaño general de los organismos; los siguientes componentes reflejan tendencias que expresan una variación de forma o proporciones.

En la fig. $4 \mathrm{~A}$ se ha representado la posición de los extremos de los vectores correspondientes a las variables en el espacio de los dos primeros componentes. Como se puede ver en esta figura, las variables pertenecientes a dife rentes órganos tienden a encontrarse en un mismo sector del espacio engendrado por los componentes; las medidas transversales $(10,16,21$ y 24$)$, por regla general, presentan una posición relativa semejante en cada grupo de variables. La distribución a lo largo de bandas de las variables correspondientes a cada órgano sugiere la existencia de gra- dientes de morfogenesis. En la fig. $4 \mathrm{~B}$ se han representado las variables en el espacio del $2^{\circ}$ y $3^{\mathrm{er}}$ componentes. Las correspondientes a medidas transversales tienden también a encontrarse agrupadas (variables 10, 16,22 y 24); entre las otras variables, las correspondientes al maxilipedo $(20,21$ y 22$)$ y antena (23 y 24) se sitúan en un sector del espacio y las correspondientes al cefalotórax $(1,2$, $3,4$ y 5$)$, furca $(8,9,10,11,12,13$ y 14) y pata $(15,16,17,18$ y 19) en otro. En el análisis realizado con el conjunto de medias de cada variable y para cada embalse (b, en la tabla I), las varianzas explicadas por los tres primeros componentes $(74,2 \%$; $7,9 \%$ y $5,0 \%$ ) son ligeramente superiores a las halladas en el análisis anterior, pero los resultados son básicamente similares.

Con los coeficientes de carga obtenidos en el análisis del conjunto de las medias (tabla I) se han calculado los valores "score" de las tres primeras componentes 
TABLA II. Valores (score) de los componentes, para el análisis realizado con las medias de las variables.

1 a Campaña

\begin{tabular}{|c|c|c|c|}
\hline Embassament & $1 \cong \mathrm{C}$ & $2 \circ \mathrm{C}$ & $3 \circ \mathrm{C}$ \\
\hline 3 & $-1,741$ & 1,255 & 0,722 \\
\hline 12 & 0,368 & $-0,482$ & $-0,703$ \\
\hline 13 & 1,325 & $-0,812$ & $-0,814$ \\
\hline 15 & 0,356 & $-1,889$ & 1,806 \\
\hline 16 & $-0,013$ & $-2,411$ & 1,817 \\
\hline 53 & 0,200 & 1,119 & 0,030 \\
\hline 56 & $-0,219$ & 1,643 & 2,280 \\
\hline 69 & $-0,282$ & 0,733 & $-0,062$ \\
\hline 72 & 0,762 & 0,296 & $-0,273$ \\
\hline 75 & $-0,003$ & 0,994 & 0,035 \\
\hline 77 & 1,965 & 0,058 & $-0,234$ \\
\hline 78 & 1,041 & 0,385 & $-0,510$ \\
\hline 79 & 0,503 & 0,451 & 0,643 \\
\hline 87 & $1,061^{\circ}$ & 0,788 & 0,205 \\
\hline 95 & 0,123 & 0,674 & 0,883 \\
\hline
\end{tabular}

para cada muestra (tabla II). Por regla general estos valores son más elevados en invierno que en verano, resultado que refleja las variaciones estacionales de tamaño de cada e mbalse.

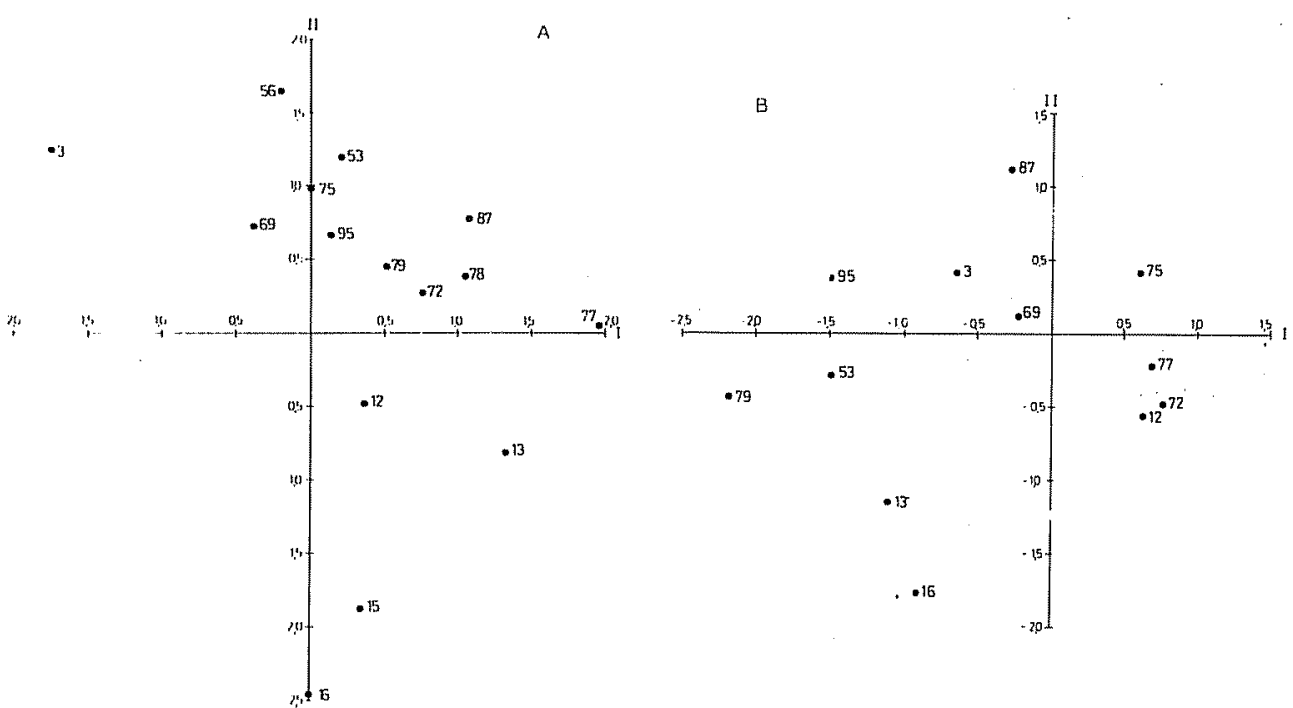

2a Campaña

$19 \mathrm{C} \quad 2 \circ \mathrm{C} \quad 3 \circ \mathrm{C}$

$\begin{array}{rrr}-0,668 & -0,417 & -0,468 \\ 0,634 & -0,573 & -0,733\end{array}$

$-1,106 \quad-1,146 \quad-1,056$

- $-1,-$

$\begin{array}{lll}-0,905 & -1,769 & 1,498\end{array}$

$-1,481 \quad-0,290 \quad-1,219$

$0, \overline{132} \quad-0, \overline{221} \quad-0, \overline{8} 60$

$0,743 \quad-0,478 \quad-1,018$

$0,605 \quad 0,410 \quad-0,303$

$0,640 \quad-0,212 \quad-0,911$

$-2, \overline{257} \quad-0, \overline{4} 58 \quad-1,386$

$-0,280 \quad 1,122 \quad 0,814$

$\begin{array}{lll}-1,500 & 0,383 & -0,184\end{array}$

En las figuras $5 \mathrm{~A} \mathrm{y} \mathrm{B}$, en las que se han representado las muestras de cada embalse utilizando como coordenadas los valores de $1^{\text {er }}$ y $2^{\circ}$ componentes del análisis realizado con las medias (b en tabla 1 ), puede verse

Figura 5. T. prasinus (hembras), representación del valor de la $1^{\text {a }}$ y $2^{\mathrm{a}}$ componente (score), con las medias de las variables de los embalses. A) $1^{\mathrm{a}}$ campaña (invierno). B) $2^{\mathrm{a}}$ campaña (verano). 
una agrupación geográfica de los embalses en función de las tendencias de variación reflejadas por estos componentes. Este resultado sugiere la existencia de razas o formas geográficas; los embalses de Galicia $(12,13,15$ y 16$)$, por ejemplo, que se ca. racterizan por unas condiciones fisicoquimicas del agua diferentes al resto de los cmbalses por su baja mineralización (MARGALEF et al. 1976), se encuentran en un mismo sector del espacio. Estas agrupaciones geograficas pueden indicar una similitud en la fenogénesis de las correspondientes poblaciones o bien pueden ser de- bidas a semejanzas de base genética que no se pueden poner de manifiesto con los métodos biométricos utilizados.

\section{AGRADECIMIENTOS}

Al Prof. Margalef por sus comentarios y ayuda en la realización del trabajo; a los compañeros del equipo de embalses del Departamento de Ecología que tomaron las muestras y a Ana $\mathrm{M}^{\mathrm{a}}$ Domingo que realizó las figuras.

\section{BIBLIOGRAFIA}

BROOKS, J.L. 1947. Turbulence as an enviromental determinant of relative growth in Daphnia Procecel.Nat. Acad. Sci, U.S.A. 33: 141-148.

CUADRAS, C.M. 1979. Mètodes đe representació de dades i la seva aplicació a la biologia. Soc. Cat. Biol. colloquis 12-13: 95-131.

COOLISY,W.W.\& P.R. LOHNI:S 1971. Multivariate data analy'sis. John Wiley \& Sons Inc. New York 364 págs.

DUSSART, B. 1969. Le's copepodes des eaux continentales d'liurope occidentale. vol. Il Boubée Paris 229 págs.

GURNI:Y, R. 1933. British fresh-water copepoda III Cy'dopoida. The Ray Soc. London $236 \mathrm{pp}$.

HUTCHINSON, G.E. 1967. A treatise un Limmolo.!. vol. II New York-London-Sydney Wiley \& Sons 1.115 págs.

JOLICOEUR, P. \& J.E. MOSSIMAN 1960. Size and shape variation in the painted turtle. A principal component analysis. Gronth 24: 339 354.

MARGALEI, R. 1947. Nota sobre algunos rotíferos. P. Inst. Biol. Apl. 4: 135-148.

1950. Segunda nota sobre la biología de las aguas estancadas del bajo Urgel. Inst. List. Ilerdenses Ilerda. 13: 329-375.

1953. Los crustace os de las aguas continentales Ibericas. Biologia de las aguas continentales; 10 Minist. Agricultura, Inst. loorest. Inves. y Exp. Madrid 245 págs.

1955. Temperatura, dimensiones y cvolución. P. Iinst. Biol. Apl. 19: $13-94$

1974. Lcologgia Omega, Barcelona 9.51 págs.

MARGALEF, R.,; PLANAS, D.; ARMENGOL, J.:
VIDAL, A.: PRAT, N; GUISET, A.; TOJA, J.; \& ESTRADA, M. 1976. Limnologia de los embalses españoles vol. I y II. Publicaciones Minist. O Públicas, Madrid. No 123.454 págs.

MIRACLI:, M.R. 1976. Distribucion en el espacio $y$ en el ticmpo de las especics del zooplancton del lago de Banyoles. Monosrafias de ICONA 5: $1-270$.

RI:YMI:NT, R.A. 1966. Afrobolinina africana, quantitative untersuchung der variabilität eincr palcozanen roraminifera. $E$. Geol. Heltr. 59: 319-338.

RI:YMFNT, R.A.\& P. SANDBI:RG 1963. Biometric study of Barramites subdifficilis. Paleontology' 6: 727-730.

RIl:RA, T. 1981. Variabilitat Morfologica de Tropocyclops i Temora. Aproximaciócritica a l'us seneralitzat de la biometria en la taxonomia de copcipodes. Tesis Univ. de Barcelona.

SI:AL, H.L. 1964, Principal components, Multivariate statistical analysis for biologist. Me thuen \& Co. $203 \mathrm{pp}$

SIIWI:LL, R.B.S. 1948. The free-swimming planktonic copepoda. Geographical distribution. The John Murray Exped. 1933-1934. Scientific Reports 8 (3): $317-592$

TONOLLI, V. 1949. Ciclo biologico, isolamento e differenziamento stagionale in popolazione naturali di un copepode abitatore di accue alpine (Aictodiapromus bacillifer) Mom litst., Ital. Idrob. 4: 95-144.

1961. Studio sulla dinamica del popolamento di un copepode (Lidiaptomus rulgaris). Mem. Inst. Ital. Idrobiol. 13: 179-202.

APENDICE: No de individuos medidos, media y error de la media para cada variable medida. El número corresponde al de la fig. 2. 


\begin{tabular}{|c|c|c|c|c|c|c|}
\hline & \multicolumn{2}{|c|}{ ALLOZ (3) } & \multicolumn{2}{|c|}{ ARBON (12) } & \multicolumn{2}{|c|}{ FORCADAS (13) } \\
\hline & $24-x-72$ & $26-V I-73$ & $23-x-72$ & $2-V I I-73$ & $29-x-72$ & $3-V I I-73$ \\
\hline 1 & $\begin{array}{l}50 \\
923,30+4,74\end{array}$ & $\begin{array}{l}50 \\
952,98+5,53\end{array}$ & $\begin{array}{l}50 \\
1014,30 \pm 4,99\end{array}$ & $\begin{array}{l}50 \\
1045,30 \pm 7,39\end{array}$ & $\begin{array}{l}50 \\
1092,00 \pm 4,05\end{array}$ & $\begin{array}{l}49 \\
919 ; 54 \pm 4,62\end{array}$ \\
\hline 2 & $\begin{array}{l}50 \\
715,25 \pm 3,48\end{array}$ & $\begin{array}{l}50 \\
736,54 \pm 4,44\end{array}$ & $\begin{array}{l}50 \\
784,42 \pm 4.27\end{array}$ & $\begin{array}{l}50 \\
301,08+0,39\end{array}$ & $\begin{array}{l}50 \\
341,92+2,54\end{array}$ & $\begin{array}{l}49 \\
712,42+3,61\end{array}$ \\
\hline 3 & $\begin{array}{l}50 \\
461,30 \pm 2,94\end{array}$ & $\begin{array}{l}50 \\
504,70 \pm 3,32\end{array}$ & $\begin{array}{l}50 \\
529,34 \pm 3,04\end{array}$ & $\begin{array}{l}50 \\
537.46 \pm 4,40\end{array}$ & $\begin{array}{l}50 \\
547,58 \pm 2,36\end{array}$ & $\begin{array}{l}49 \\
502,35 \pm 3,37\end{array}$ \\
\hline 4 & $\begin{array}{l}50 \\
310,6 \overline{ \pm}+2,39\end{array}$ & $\begin{array}{l}50 \\
324,24 \pm 1,89\end{array}$ & $\begin{array}{l}50 \\
350,70 \pm 2,39\end{array}$ & $\begin{array}{l}49 \\
350,57 \pm 3,04\end{array}$ & $\begin{array}{l}50 \\
358,53+2,37\end{array}$ & $\begin{array}{l}48 \\
326,40+2,33\end{array}$ \\
\hline 5 & $\begin{array}{l}50 \\
232,40 \pm 1,73\end{array}$ & $\begin{array}{l}50 \\
240,66 \pm 1,36\end{array}$ & $\begin{array}{l}50 \\
256,34+1,38\end{array}$ & $\begin{array}{l}50 \\
266,98+2,52\end{array}$ & $\begin{array}{l}50 \\
277,76 \pm 4,31\end{array}$ & $\begin{array}{l}49 \\
244,00+1,35\end{array}$ \\
\hline 3 & $\begin{array}{l}50 \\
49,55 \pm 0,38\end{array}$ & $\begin{array}{l}50 \\
52,35 \pm 0,32\end{array}$ & $\begin{array}{l}50 \\
55,05 \pm 0,39\end{array}$ & $\begin{array}{l}50 \\
57,00 \pm 1,72\end{array}$ & $\begin{array}{l}50 \\
59,77+0,35\end{array}$ & $\begin{array}{l}49 \\
50,79+10,31\end{array}$ \\
\hline 3 & $\begin{array}{l}50 \\
20,37 \pm 0,22\end{array}$ & $\begin{array}{l}50 \\
22,57 \pm 0,23\end{array}$ & $\begin{array}{l}50 \\
23,37 \pm 0,20\end{array}$ & $\begin{array}{l}50 \\
23,57 \pm 0,28\end{array}$ & $\begin{array}{l}50 \\
25,12+0, i 6\end{array}$ & $\begin{array}{l}49 \\
21,68+0,22\end{array}$ \\
\hline 10 & $\begin{array}{l}50 \\
20,30+0,25\end{array}$ & $\begin{array}{l}50 \\
22,15 \pm 0,20\end{array}$ & $\begin{array}{l}50 \\
22,35 \pm 0,21\end{array}$ & $\begin{array}{l}50 \\
22,07 \pm 0,23\end{array}$ & $\begin{array}{l}50 \\
24,30 \pm 0,27\end{array}$ & $\begin{array}{l}49 \\
22,57 \pm 0,2=\end{array}$ \\
\hline 11 & $\begin{array}{l}50 \\
33,37 \pm 0,41\end{array}$ & $\begin{array}{l}50 \\
35,77 \pm 0,32\end{array}$ & $\begin{array}{l}50 \\
41,35 \pm 0,41\end{array}$ & $\begin{array}{l}50 \\
12,42 \div 0,41\end{array}$ & $\begin{array}{l}50 \\
43,05 \pm 0,42\end{array}$ & $\begin{array}{l}49 \\
37,4 a+0,38\end{array}$ \\
\hline 12 & $\begin{array}{l}48 \\
145,41 \pm 1,03\end{array}$ & $\begin{array}{l}50 \\
152,77 \pm 0,73\end{array}$ & $\begin{array}{l}50 \\
161,65+0, \geqslant 9\end{array}$ & $\begin{array}{l}50 \\
i 62.42 \pm 1,29\end{array}$ & $\begin{array}{l}30 \\
169,70+0,35\end{array}$ & $\begin{array}{l}19 \\
=43,26+1,00\end{array}$ \\
\hline \pm 3 & $\begin{array}{l}49 \\
222,14 \pm 1,85\end{array}$ & $\begin{array}{l}49 \\
241,32 \pm 1,23\end{array}$ & $\begin{array}{l}\Delta 9 \\
251,71+1,56\end{array}$ & $\begin{array}{l}50 \\
250,70 \pm 1,97\end{array}$ & $\begin{array}{l}50 \\
258,75 \pm 1,53\end{array}$ & $\begin{array}{l}48 \\
221, \pm 4 \pm 1,47\end{array}$ \\
\hline$i 4$ & $\begin{array}{l}50 \\
36,37 \pm 0,37\end{array}$ & $\begin{array}{l}30 \\
41,12 \pm 0,29\end{array}$ & $\begin{array}{l}50 \\
42,32+0,36\end{array}$ & 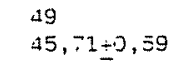 & $\begin{array}{l}50 \\
44,35+0,47\end{array}$ & $47,67-5,48$ \\
\hline 15 & $\begin{array}{l}48 \\
42,38+0,29\end{array}$ & 50 & $\begin{array}{l}50 \\
45,35+0,24\end{array}$ & $\begin{array}{l}50 \\
44,57+0,20\end{array}$ & $\begin{array}{l}49 \\
47,24 \pm 0,29\end{array}$ & $\begin{array}{l}1.3 \\
40,49+0,23\end{array}$ \\
\hline-5 & $\begin{array}{l}48 \\
22,31=0,19\end{array}$ & $\begin{array}{l}50 \\
13,92 \pm 1,24\end{array}$ & $\begin{array}{l}50 \\
14,37 \div 0,15\end{array}$ & $\begin{array}{l}50 \\
14,32+0,10\end{array}$ & $\begin{array}{l}49 \\
14,59+0,22\end{array}$ & $\begin{array}{l}48 \\
13,56 \pm 0,1^{-}\end{array}$ \\
\hline 17 & 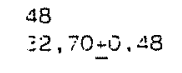 & $\begin{array}{l}18 \\
35,34+0,33\end{array}$ & $\begin{array}{l}50 \\
58,50+0,59\end{array}$ & $\begin{array}{l}50 \\
50,50+0,37 .\end{array}$ & $\frac{19}{73}, 67+0,10$ & $\begin{array}{l}43 \\
03,20+0,32\end{array}$ \\
\hline 18 & $\begin{array}{l}48 \\
25,38 \div 0,33\end{array}$ & $\begin{array}{l}43 \\
27,08+17,25\end{array}$ & $\begin{array}{l}50 \\
29,77+0,20\end{array}$ & $\begin{array}{l}50 \\
30,17 \pm 0,30\end{array}$ & $\begin{array}{l}45 \\
32,24 \pm 0,45\end{array}$ & $\begin{array}{l}4.3 \\
23,02+0,3=\end{array}$ \\
\hline$\therefore 9$ & $\begin{array}{l}48 \\
74,97 \pm 0,58\end{array}$ & $\begin{array}{l}49 \\
79,33+0,49\end{array}$ & $\begin{array}{l}50 \\
30,47 \pm 0,65\end{array}$ & $\begin{array}{l}50 \\
31,72 \pm 0,31\end{array}$ & $\begin{array}{l}19 \\
55,57+0,57\end{array}$ & $\begin{array}{l}48 \\
70,19+0,11\end{array}$ \\
\hline 20 & $\begin{array}{l}44 \\
43,49 \pm 0,45\end{array}$ & $\begin{array}{l}13 \\
50,13 \pm 0,25\end{array}$ & $\begin{array}{l}43 \\
52,7 \pm 0,25\end{array}$ & $\begin{array}{l}49 \\
52,: 6+0,35\end{array}$ & $\begin{array}{l}39 \\
52,73+0,30\end{array}$ & $\begin{array}{l}45 \\
47.03+0,34\end{array}$ \\
\hline 21 & $\begin{array}{l} \pm 4 \\
18,41 \pm 0,23\end{array}$ & $\begin{array}{l} \pm 3 \\
\pm 5,30+12,19\end{array}$ & $\begin{array}{l} \pm 8 \\
\pm 9,48+1,16\end{array}$ & $\begin{array}{l}49 \\
19,13+1), 24\end{array}$ & $\begin{array}{l}39 \\
19,30+0,22\end{array}$ & $\begin{array}{l}45 \\
13,52+0,2-\end{array}$ \\
\hline 22 & $\begin{array}{l}15 \\
: 3,34+6,25\end{array}$ & $\begin{array}{l} \pm 8 \\
: 9,3 Q_{ \pm 0}, 13\end{array}$ & $\begin{array}{l} \pm 8 \\
: 9.35 \pm 19,24\end{array}$ & $\begin{array}{l}47 \\
29,32-2,24\end{array}$ & $\begin{array}{l}36 \\
30, \therefore 6+0,37\end{array}$ & $\begin{array}{l}15 \\
13,38+0,1=\end{array}$ \\
\hline 23 & $\begin{array}{l}43 \\
.5, \exists 3+0,35\end{array}$ & $\begin{array}{l}50 \\
51,77+0,30\end{array}$ & $\begin{array}{l}50 \\
53,37-1), 33\end{array}$ & $\begin{array}{l}50 \\
55, i 7-0,30\end{array}$ & $\begin{array}{l}59 \\
56,31=0,31\end{array}$ & $\begin{array}{l}19 \\
50,30+3.35\end{array}$ \\
\hline 24 & $\begin{array}{l}12 \\
\therefore \therefore, 25-0,13\end{array}$ & $\begin{array}{l}50 \\
12,47+0,14\end{array}$ & $\begin{array}{l}50 \\
13,20+0,15\end{array}$ & $\begin{array}{l}30 \\
i 3,37+3,21\end{array}$ & $\begin{array}{l}19 \\
13,22-0,15\end{array}$ & $\begin{array}{l}19 \\
13,23+1,23\end{array}$ \\
\hline
\end{tabular}




\begin{tabular}{|c|c|c|c|c|c|c|}
\hline & PORTO DE & UROS $(16)$ & GUA & RAZ (53) & RETOR & ILLO (69) \\
\hline & $30-x-72$ & $5-$ VII -73 & $12-X I I-72$ & $17-V I I-73$ & $23-1-73$ & $15-V-73$ \\
\hline 1 & $\begin{array}{l}50 \\
1005,62 \pm 3,21\end{array}$ & $\begin{array}{l}50 \\
947,24 \pm 3,69\end{array}$ & $\begin{array}{l}50 \\
994,28+3,74\end{array}$ & $\begin{array}{l}50 \\
905,24 \pm 5,60\end{array}$ & $\begin{array}{l}50 \\
992,32 \pm 5,35\end{array}$ & $\begin{array}{l}50 \\
1011,50 \pm 5,89\end{array}$ \\
\hline 2 & $\begin{array}{l}50 \\
733,30 \pm 2,45\end{array}$ & $\begin{array}{l}50 \\
740,04 \pm 2,88\end{array}$ & $\begin{array}{l}50 \\
754,32+2,72\end{array}$ & $\begin{array}{l}50 \\
711,20+4,44\end{array}$ & $\begin{array}{l}50 \\
755,72 \pm 4,18\end{array}$ & $\begin{array}{l}50 \\
778,54+4,33\end{array}$ \\
\hline 3 & $\begin{array}{l}50 \\
508,76 \pm 1,97\end{array}$ & $\begin{array}{l}48 \\
505,75 \pm 2,83\end{array}$ & $\begin{array}{l}50 \\
495,32+2,09\end{array}$ & $\begin{array}{l}50 \\
468,58 \pm 3,30\end{array}$ & $\begin{array}{l}50 \\
512,12+3,05\end{array}$ & $\begin{array}{l}50 \\
520,66 \pm 3,45\end{array}$ \\
\hline 4 & $\begin{array}{l}50 \\
348,32 \pm 1,73\end{array}$ & $\begin{array}{l}50 \\
335,72 \pm 1,77\end{array}$ & $\begin{array}{l}50 \\
344,96 \pm 1,92\end{array}$ & $\begin{array}{l}49 \\
319,28 \pm 1,96\end{array}$ & $\begin{array}{l}50 \\
331,66 \pm 2,61\end{array}$ & $\begin{array}{l}50 \\
344,96+1,86\end{array}$ \\
\hline 5 & $\begin{array}{l}50 \\
257,60 \pm 1,13\end{array}$ & $\begin{array}{l}50 \\
251,30 \pm 1,08\end{array}$ & $\begin{array}{l}50 \\
259,00 \pm 1,03\end{array}$ & $\begin{array}{l}50 \\
240,24 \pm 1,37\end{array}$ & $\begin{array}{l}50 \\
246,40+1,77\end{array}$ & $\begin{array}{l}50 \\
259,28 \pm 1,32\end{array}$ \\
\hline 8 & $\begin{array}{l}50 \\
53,10 \div 0,29\end{array}$ & $\begin{array}{l}50 \\
50,07+0,30\end{array}$ & $\begin{array}{l}50 \\
54,87 \pm 0,24\end{array}$ & $\begin{array}{l}50 \\
51,45 \pm 0,27\end{array}$ & $\begin{array}{l}50 \\
53,57 \pm 0,33\end{array}$ & $\begin{array}{l}50 \\
55,30+0,34\end{array}$ \\
\hline 9 & $\begin{array}{l}50 \\
23,37 \pm 0,20\end{array}$ & $\begin{array}{l}50 \\
21,40 \pm 0,20\end{array}$ & $\begin{array}{l}50 \\
22,45+0,15\end{array}$ & $\begin{array}{l}50 \\
21,30+0,20\end{array}$ & $\begin{array}{l}50 \\
22,65 \pm 0,24\end{array}$ & $\begin{array}{l}50 \\
22,82+0,17\end{array}$ \\
\hline 10 & $\begin{array}{l}50 \\
24,30 \pm 0,25\end{array}$ & $\begin{array}{l}50 \\
22,20+0,25\end{array}$ & $\begin{array}{l}50 \\
21,92+0,21\end{array}$ & $\begin{array}{l}50 \\
22,60 \pm 0,22\end{array}$ & $\begin{array}{l}50 \\
21,45 \pm 0,21\end{array}$ & $\begin{array}{l}50 \\
23,20 \pm 0,24\end{array}$ \\
\hline 11 & $\begin{array}{l}50 \\
39,65+0,29\end{array}$ & $\begin{array}{l}50 \\
35,65+0,35\end{array}$ & $\begin{array}{l}50 \\
38,57 \pm 0,29\end{array}$ & $\begin{array}{l}50 \\
34,00+0,33\end{array}$ & $\begin{array}{l}50 \\
39,00+0,39\end{array}$ & $\begin{array}{l}50 \\
38,62+0,33\end{array}$ \\
\hline 12 & $\begin{array}{l}50 \\
164,50+0,72\end{array}$ & $\begin{array}{l}50 \\
155,37 \pm 0,71\end{array}$ & $\begin{array}{l}50 \\
161,95 \pm 1,11\end{array}$ & $\begin{array}{l}50 \\
139,65 \pm 0,97\end{array}$ & $\begin{array}{l}50 \\
1.58,12+0,94\end{array}$ & $\begin{array}{l}50 \\
156,25+0,88\end{array}$ \\
\hline 13 & $\begin{array}{l}49 \\
233,47+0,94\end{array}$ & $\begin{array}{l}50 \\
218,00 \pm 1,29\end{array}$ & $\begin{array}{l}50 \\
257,85 \pm 1,47\end{array}$ & $\begin{array}{l}49 \\
211,02+1,25\end{array}$ & $\begin{array}{l}49 \\
257,24+1,89\end{array}$ & $\begin{array}{l}49 \\
244,31 \pm 1,69\end{array}$ \\
\hline 14 & $\begin{array}{l}50 \\
52,55+0,52\end{array}$ & $\begin{array}{l}50 \\
50,42 \pm 0,48\end{array}$ & $\begin{array}{l}50 \\
41,87 \pm 0,56\end{array}$ & $\begin{array}{l}50 \\
37,55 \pm 0,32\end{array}$ & $\begin{array}{l}50 \\
41,15 \pm 0,26\end{array}$ & $\begin{array}{l}49 \\
42,19 \pm 0,31\end{array}$ \\
\hline 15 & $\begin{array}{l}49 \\
45,30+0,24\end{array}$ & $\begin{array}{l}50 \\
41,80 \pm 0,24\end{array}$ & $\begin{array}{l}50 \\
46,30+0,20\end{array}$ & $\begin{array}{l}50 \\
40,07 \pm 0,27\end{array}$ & $\begin{array}{l}49 \\
44,38 \pm 0,30\end{array}$ & $\begin{array}{l}50 \\
43,40 \pm 0,25\end{array}$ \\
\hline 16 & $\begin{array}{l}49 \\
13,49 \pm 0,17\end{array}$ & $\begin{array}{l}50 \\
12,85 \pm 0,15\end{array}$ & $\begin{array}{l}50 \\
14,15 \pm 0,13\end{array}$ & $\begin{array}{l}50 \\
13,47 \pm 0,16\end{array}$ & $\begin{array}{l}49^{\circ} \\
13,52 \pm 0,16\end{array}$ & $\begin{array}{l}50 \\
13,92 \pm 0,13\end{array}$ \\
\hline 17 & $\begin{array}{l}49 \\
73,36+0,41\end{array}$ & $\begin{array}{l}49 \\
68,01 \pm 0,40\end{array}$ & $\begin{array}{l}50 \\
67,27 \pm 0,41\end{array}$ & $\begin{array}{l}50 \\
60,60+0,45\end{array}$ & $\begin{array}{l}49 \\
65,05 \pm 0,54\end{array}$ & $\begin{array}{l}50 \\
67,00+0,38\end{array}$ \\
\hline 18 & $\begin{array}{l}49 \\
23,08+0,28\end{array}$ & $\begin{array}{l}50 \\
27,35 \pm 0,23\end{array}$ & $\begin{array}{l}50 \\
29,92+0,27\end{array}$ & $\begin{array}{l}50 \\
27,85 \pm 0,32\end{array}$ & $\begin{array}{l}49 \\
27,52+0,33\end{array}$ & $\begin{array}{l}50 \\
29,35 \pm 0,27\end{array}$ \\
\hline 19 & $\begin{array}{l}49 \\
73,11+0,40\end{array}$ & $\begin{array}{l}50 \\
70,80+0,61\end{array}$ & $\begin{array}{l}50 \\
87,32 \pm 0,68\end{array}$ & $\begin{array}{l}50 \\
66,35+0,53\end{array}$ & $\begin{array}{l}49 \\
86,86 \pm 0,73\end{array}$ & $\begin{array}{l}50 \\
80,12 \pm 0,76\end{array}$ \\
\hline 20 & $\begin{array}{l}47 \\
49,57 \pm 0,32\end{array}$ & $\begin{array}{l}47 \\
48,16+0,26\end{array}$ & $\begin{array}{l}48 \\
53,17 \pm 0,29\end{array}$ & $\begin{array}{l}47 \\
48,46 \pm 0,23\end{array}$ & $\begin{array}{l}48 \\
51,04 \pm 0,32\end{array}$ & $\begin{array}{l}49 \\
51,83+0,25\end{array}$ \\
\hline 21 & $\begin{array}{l}47 \\
19,07 \pm 0,20\end{array}$ & $\begin{array}{l}47 \\
18,59+0,21\end{array}$ & $\begin{array}{l}48 \\
20,02+0,16\end{array}$ & $\begin{array}{l}47 \\
18,51 \pm 0,16\end{array}$ & $\begin{array}{l}49 \\
19,74+0,18\end{array}$ & $\begin{array}{l}49 \\
19,77+0,15\end{array}$ \\
\hline 22 & $\begin{array}{l}45 \\
18,52 \pm 0,24\end{array}$ & $\begin{array}{l}46 \\
18,77 \pm 0,18\end{array}$ & $\begin{array}{l}47 \\
19,76+0,16\end{array}$ & $\begin{array}{l}47 \\
18,51 \pm 0,16\end{array}$ & $\begin{array}{l}49 \\
18,87 \pm 0,22\end{array}$ & $\begin{array}{l}48 \\
19,58 \pm 0,16\end{array}$ \\
\hline 23 & $\begin{array}{l}49 \\
49,21 \pm 0,20\end{array}$ & $\begin{array}{l}48 \\
48,07 \pm 0,24\end{array}$ & $\begin{array}{l}50 \\
54,25+0,26\end{array}$ & $\begin{array}{l}49 \\
50,43 \pm 0,26\end{array}$ & $\begin{array}{l}50 \\
51,85+0,31\end{array}$ & $\begin{array}{l}50 \\
53,95+0,27\end{array}$ \\
\hline 24 & $\begin{array}{l}49 \\
12,35+0,20\end{array}$ & $\begin{array}{l}48 \\
12,65+0,17\end{array}$ & $\begin{array}{l}50 \\
12,67+0,11\end{array}$ & $\begin{array}{l}49 \\
12,60+0,14\end{array}$ & $\begin{array}{l}50 \\
12,77+0,15\end{array}$ & $\begin{array}{l}50 \\
12,95+0,15\end{array}$ \\
\hline
\end{tabular}




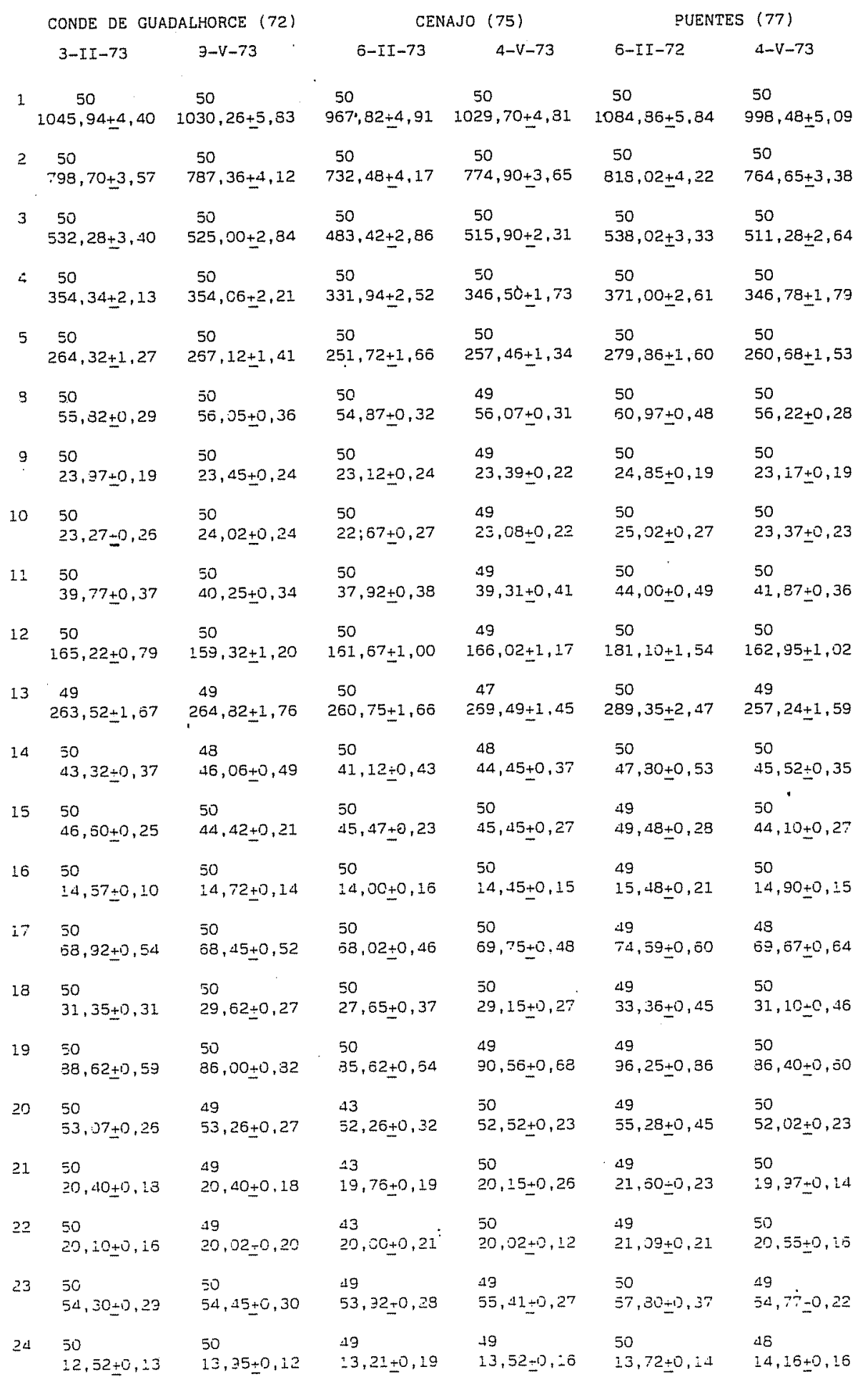




\begin{tabular}{|c|c|c|c|c|c|}
\hline \multicolumn{2}{|c|}{ BOAOELLA 1791} & \multicolumn{2}{|c|}{ SCRNOS $(37)$} & \multicolumn{2}{|c|}{ OLIANA } \\
\hline $24-1=I-73$ & $9-V I I \bar{i}-73$ & $31-1-73$ & $22-\operatorname{VIII}-73$ & $19-i I I-73$ & $7-Y I I Z-7 z$ \\
\hline 50 & 50 & 50 & 50 & 50 & 50 \\
\hline $1019,48_{i} 3,45$ & $384,30 \pm 3,31$ & $1067,50+7,90$ & $396,24 \pm 5,47$ & $998,75 \pm 3,46$ & $927,38+4,06$ \\
\hline 50 & 50 & 50 & 50 & 50 & 50 \\
\hline $772,38 \pm 2,76$ & $520,42 \div 2,50$ & $303,24+6,: 3$ & $748,36 \pm 3,58$ & $755,15 \pm 2,37$ & $=10,20 \pm 3,34$ \\
\hline 50 & 30 & 50 & 50 & 30 & 49 \\
\hline $516,04 \pm 2,95$ & $473,34+2,37$ & $540,25 \pm 4,29$ & $491,32 \div 2,58$ & $503,30+2,77$ & $485,42 \pm 2,37$ \\
\hline 50 & 50 & 50 & 50 & Eo & 49 \\
\hline $344,25+1,05$ & $315,38+1,38$ & $359,10+3,30$ & $343,42 \div 1,46$ & $330,28 \pm 2,17$ & $327.00 \pm 1.70$ \\
\hline 50 & 50 & 50 & 50 & 50 & 50 \\
\hline $260,26 \pm 0,97$ & $239,58 \pm 1,25$ & $253,34 \pm 2,19$ & $260,54 \pm 1,18$ & $258,56 \pm 1,36$ & $248,54 \pm i, 22$ \\
\hline 50 & so & 50 & 53 & 50 & 50 \\
\hline $56,50+0,67$ & $46,35+0,29$ & $58, \equiv 3,4 \equiv \pm 0,23$ & $34,30=0,33$ & $53,75+0,28$ & $50,45 \pm 0,25$ \\
\hline$\Xi 0$ & 50 & 50 & 50 & 50 & 30 \\
\hline $23,00 \div 19,17$ & $19,30 \pm 19,24$ & $23,70 \div 0,31$ & $22,45 \pm 0,24$ & $22,52+0,21$ & $20,55+2,16$ \\
\hline 50 & 50 & 50 & 50 & 50 & 50 \\
\hline $21,50 \pm 0,29$ & $21,25+0,23$ & $21,30+12,29$ & $20,02 \div 0.22$ & $21,70 \pm 0,26$ & $20,35 \pm 10,22$ \\
\hline 50 & 50 & 50 & 50 & 50 & 50 \\
\hline $42,20 \pm 0,32$ & $31,32 \pm 0,18$ & $43,23+1,=5$ & $38,32 \pm 0,35$ & $39,27=3,29$ & $35,30 \pm 0,29$ \\
\hline 50 & 49 & 50 & 50 & 19 & 19 \\
\hline$: 67,55 \pm 0,95$ & $134,46 \pm 0,70$ & $175,90 \pm 1,53$ & $159,17 \pm 1,03$ & $165,38 \pm 0,83$ & $: 45,53+0,75$ \\
\hline 50 & 50 & 50 & $\equiv 0$ & $49^{\circ}$ & 50 \\
\hline $259.55 \pm 1,40$ & $211,22 \pm 0,38$ & $287,32 \pm 2,48$ & $250,10 \pm 1,37$ & $253,52 \pm 1,55$ & $230,92 \pm 1,12$ \\
\hline 50 & 50 & 50 & 47 & 50 & 49 \\
\hline $47,35 \pm 0,32$ & $38,02 \pm 0,34$ & $47,37=0,32$ & $43,03+0,37$ & $46,32+0,39$ & $39,39 \pm 0,21$ \\
\hline 49 & 10 & 50 & 49 & 50 & 19 \\
\hline $46,03+1,25$ & $38,35+0,19$ & $47.15+0,25$ & $43,13+0,24$ & $45, .5+0,17$ & $30,38 \pm 0,23$ \\
\hline 49 & 49 & 50 & 49 & 50 & 19 \\
\hline $13,90+0,12$ & $13,57=0,13$ & $i 4,27=0,20$ & $: 2,38 \pm 13,: 3$ & $13, \equiv 2+0, \therefore 3$ & $: 2,57=0,: 3$ \\
\hline 19 & 40 & 50 & 49 & 50 & 19 \\
\hline $59,13 \pm 0,46$ & $50,48+0,39$ & $20.02+0,52$ & $65,34=2,45$ & $57,25+5,10$ & $51,52=0,45$ \\
\hline 49 & 19 & 50 & 18 & 50 & 43 \\
\hline $20,11+0,30$ & $24,18+0,35$ & $28,70+0,37$ & $23,32 \pm 0,23$ & $20,45 \pm 0,30$ & $27,52+1,-11$ \\
\hline 19 & 49 & 50 & 49 & $4 \subseteq$ & 10 \\
\hline $90,31 \pm 0,0 \overline{0}$ & $55,32 \div 12,42$ & $36,27=1,10$ & $31,5 \pm+0,59$ & $99,33 \pm 0,52$ & $72,29 \pm 0,04$ \\
\hline 17 & \pm 9 & $\$ 1$ & 19 & 17 & $1 a$ \\
\hline $52,13 \pm 0,29$ & $40,3+2,25$ & $53, \equiv 6 \div 1,32^{\circ}$ & $52,22 \pm 0,25$ & $31,46 \pm, 22$ & $\pm 7,57 \pm 0,23$ \\
\hline 47 & 49 & $1 \mathrm{i}$ & 50 & 4. & Itis \\
\hline $19,31 \pm 0,15$ & $1 \div, 55+0,15$ & $20,30 \pm 0,27$ & $15,-7=2, \pm 5$ & $\therefore 3,34 \pm 0,23$ & $\therefore 3,23=3,20$ \\
\hline 47 & 10 & 40 & 50 & 17 & $2 \exists$ \\
\hline$i g, z 5+0, i z$ & $17.05 \pm 0,10$ & $20,53=0,20$ & $: 2,32 \pm 0, \pm 2$ & $\therefore 9,30, \pm$. & $\therefore 3,49 \pm 1, \therefore 3$ \\
\hline 50 & $\therefore 9$ & 19 & 50 & 50 & $\Xi 9$ \\
\hline $34,57+1,20$ & $47,39+0,23$ & 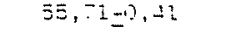 & $32.30+3.27$ & $53.25-2,21$ & $30,2=2.20$ \\
\hline 50 & \pm 9 & 19 & $\Xi i$ & $\Xi 0$ & 50 \\
\hline $13, \pm 5 \pm 1), 14$ & $12,39+0,10$ & $13,13 \div 0, \pm 5$ & $\pm 2,55 \pm 2 . \therefore$ & $\therefore 2,55+13, \pm 5$ & $\therefore,^{-\infty}=2, \therefore 1$ \\
\hline
\end{tabular}

